Provided for non-commercial research and education use. Not for reproduction, distribution or commercial use.

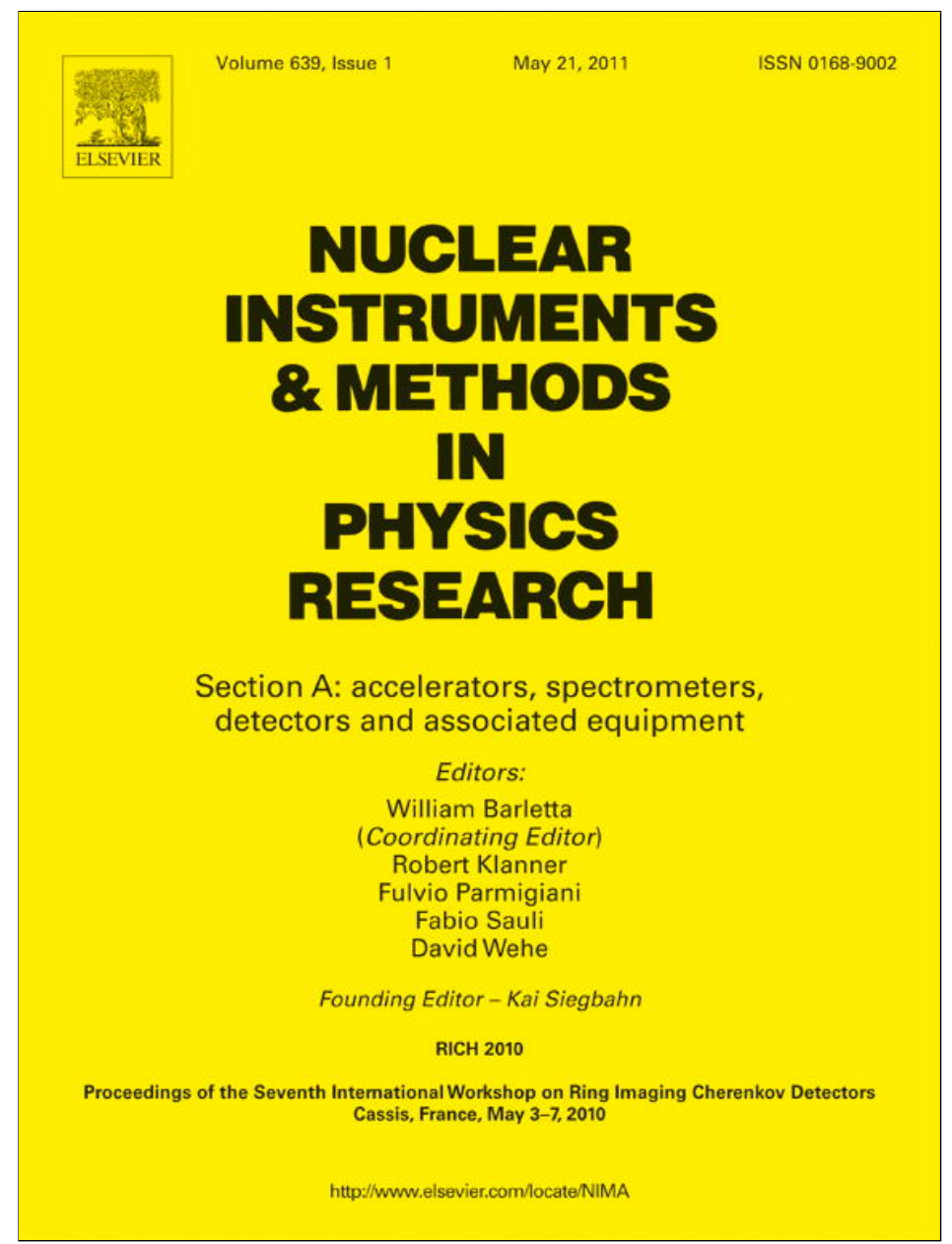

This article appeared in a journal published by Elsevier. The attached copy is furnished to the author for internal non-commercial research and education use, including for instruction at the authors institution and sharing with colleagues.

Other uses, including reproduction and distribution, or selling or licensing copies, or posting to personal, institutional or third party websites are prohibited.

In most cases authors are permitted to post their version of the article (e.g. in Word or Tex form) to their personal website or institutional repository. Authors requiring further information regarding Elsevier's archiving and manuscript policies are encouraged to visit:

http://www.elsevier.com/copyright 


\title{
The NA62 RICH detector
}

\author{
Massimo Lenti \\ Sezione dell'INFN di Firenze, I-50019 Sesto Fiorentino, Italy
}

On behalf of the NA62 Collaboration

\section{A R T I C L E I N F O}

\section{Available online 1 September 2010}

Keywords:

$\mathrm{RICH}$

PID

Timing

\begin{abstract}
A B S T R A C T
The NA62 RICH must separate charged pions from muons in a $15-35 \mathrm{GeV} / \mathrm{c}$ momentum range. The detector will have a $17 \mathrm{~m}$ long, $4 \mathrm{~m}$ wide cylindrical vessel, filled with neon at an atmospheric pressure. A mosaic of 20 mirrors, with $17 \mathrm{~m}$ focal length will be placed at the downstream end of the vessel while 2000 single anode photomultipliers will be placed at the upstream part. Two test beams were held at CERN in 2007 and 2009 with a RICH prototype. A muon suppression factor greater than 100 was validated.
\end{abstract}

(c) 2010 Elsevier B.V. All rights reserved.

\section{Introduction}

The CERN NA62 experiment [1] aims at a 10\% measurement of the ultra-rare decay $K^{+} \rightarrow \pi^{+} v \bar{v}$, predicted with high precision to be $(0.85 \pm 0.07) \times 10^{-10}$ in the standard model. All the other kaon decays are background for this measurement and the most important channel is $K^{+} \rightarrow \mu^{+} \nu$ which can be suppressed by kinematical cuts and from the different penetrating power of muons with respect to pions. A further suppression factor of more than 100 can be accomplished with particle identification given by a RICH detector. The momentum range for pion-muon separation is between 15 and $35 \mathrm{GeV} / c$ and neon gas at the atmospheric pressure as Cherenkov radiating medium has a pion threshold at $12 \mathrm{GeV} / c$. Neon is characterized by a very low chromatic dispersion but its small index of refraction requires to have a long radiating medium to produce enough photons: in the NA62 layout there will be about $18 \mathrm{~m}$ between the last chamber of the magnetic spectrometer and the electromagnetic calorimeter, which can be used to accomodate a RICH detector. The RICH must also have a time resolution of about $100 \mathrm{ps}$ to disentangle accidental associations of beam particles seen by the beam spectrometer $(750 \mathrm{MHz}$ rate) with pions, coming from kaon decays, detected by the spectrometer $(10 \mathrm{MHz}$ rate): fast single anode photomultipliers (PMT) are needed. The 2000 PMTs will be arranged into two groups to avoid the shadow induced by a beam pipe where undecayed beam particles will pass; a PMT interdistance of $18 \mathrm{~mm}$ is enough to reach the requested Cherenkov angle resolution (Fig. 1).

\section{Vessel and gas system}

The RICH vessel must contain the neon gas without leaks, supporting a maximum overpressure of 150 mbar with respect to atmospheric pressure. The constraints to be matched are the available space for the access shaft to the experimental cavern, the transverse fiducial region for $\gamma$ from $\pi^{0}$ decays both upstream and downstream of the detector, and the space needed to put 2000 PMTs. A $17 \mathrm{~m}$ long cylindrical vessel, made of construction steel, divided into four sections of decreasing diameter, $4 \mathrm{~m}$ wide at the upstream part and $3.4 \mathrm{~m}$ wide at the downstream end, is envisaged. The upstream window, made of thin aluminium, will be in direct contact with the decay vacuum tank; the thin aluminium downstream window will be in contact with air. The vessel will be tilted by $2.4 \mathrm{mrad}$ in the horizontal plane to follow the path of undecayed charged beam particles passing through a $157 \mathrm{~mm}$ diameter beam pipe. The vessel volume will be about $200 \mathrm{~m}^{3}$.

The neon gas will be kept slightly above the atmospheric pressure with a density stability better than $1 \%$ and a contaminant level smaller than $1 \%$. The vessel will be first filled with an inexpensive $\mathrm{CO}_{2}$ and then the gas will be circulated in a closed loop introducing neon and removing $\mathrm{CO}_{2}$, keeping the pressure almost constant. When the $\mathrm{CO}_{2}$ content will be below the desired level the gas loop will be terminated and the vessel will be valve closed.

\section{Mirrors}

A mosaic of 20 mirrors will be used to reflect the Cherenkov light, focusing it onto the PMTs location. The 18 mirrors of
E-mail address: lenti@fi.infn.it 


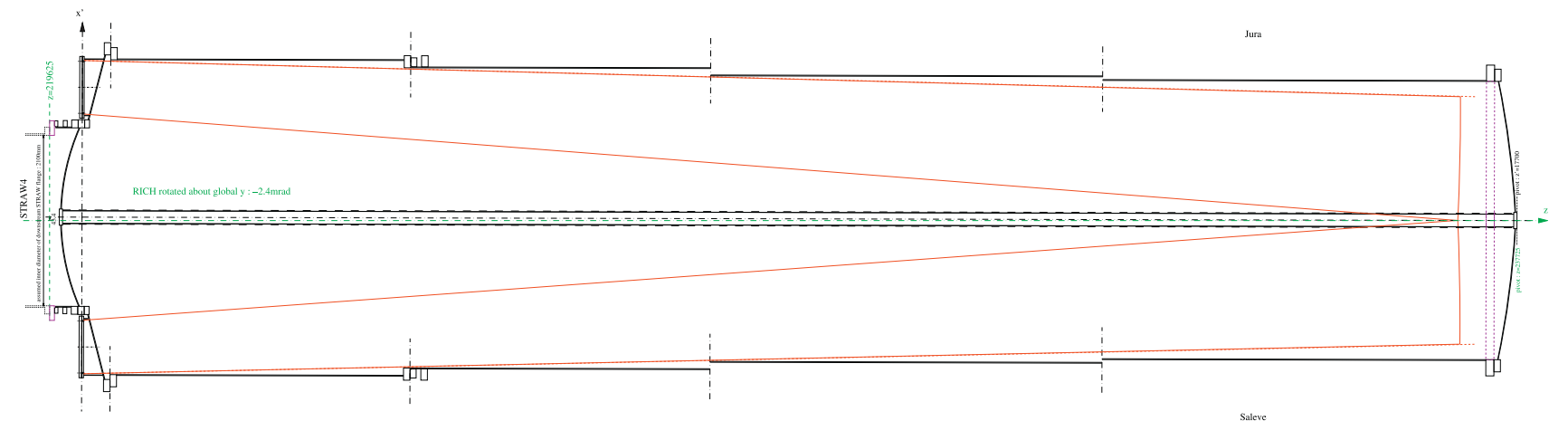

Fig. 1. Top view of the NA62 RICH detector. The beam comes from the left; optical paths from the mirrors to the PMTs are shown.

hexagonal shape and two semihexagonal ones will be used. Each mirror is $700 \mathrm{~mm}$ wide at maximum, $25 \mathrm{~mm}$ thick and made with glass; the semihexagonal mirrors have a $90 \mathrm{~mm}$ radius semi-hole on the long side because they will be placed around the beam pipe. The optical surface will be coated with a thin aluminium layer to improve the UV reflectivity, covered with a dielectric film to avoid oxidation. The mirrors must have $17 \mathrm{~m}$ focal length with a $D_{0}$ quality parameter around $1 \mathrm{~mm}\left(D_{0}\right.$ is the diameter of the minimum circle where $95 \%$ of the light coming from a point source placed in the mirror center of curvature is imaged). On the non-reflecting surface of each mirror two holes will be drilled along the barycenter line $540 \mathrm{~mm}$ apart for the hexagonal shape, $70 \mathrm{~mm}$ for the semihexagonal one. Each mirror will be hung inserting in each hole a dowel connected to a supporting wall. Piezo-electric actuators will be used for mirror alignment both in the vertical and horizontal directions. Particular care will be used to minimize the material in front of the downstream electromagnetic calorimeter.

\section{Photomultipliers and readout}

Fast single anode PMTs will be used and the Hamamatsu R7400U-03 has been chosen. It has a UV-glass entrance window and a bialkali photocathode which provide a photon response between 185 and $650 \mathrm{~nm}$ with a peak at $420 \mathrm{~nm}$ where the quantum efficiency is about $20 \%$. The PMT has 280 ps transit time jitter (FWHM) and operated at $900 \mathrm{~V}$ negative voltage provides a single photon output signal of $240 \mathrm{fC}$ on average $\left(1.5 \times 10^{6}\right.$ average gain with eight dynodes). The PMT transverse size is $16 \mathrm{~mm}$ with a minimum active diameter of $8 \mathrm{~mm}$. The Cherenkov light will be collected to the PMT active region with a Winston (truncated) cone $18 \mathrm{~mm}$ wide at the beginning, $7.5 \mathrm{~mm}$ wide in front of the PMT, $22 \mathrm{~mm}$ high, covered with aluminized mylar to improve reflectivity. Particular care will be taken to remove the $0.3 \mathrm{~W}$ of heat that each PMT produces.

The NINO ASIC [2] will be used as a fast discriminator operated in time-over-threshold mode. This ASIC has been chosen for its intrinsic time resolution better than $50 \mathrm{ps}$. The LVDS output of the discriminator will be sent to an HPTDC embedded on a TELL1 board [3]. Each board will have 512 channels. The RICH will be used in the first level trigger.

\section{Results from prototype test beams}

To validate the RICH project a prototype was built, with a $17 \mathrm{~m}$ long, $0.6 \mathrm{~m}$ wide cylindrical vessel, vacuum proof. A $17 \mathrm{~m}$ focal length, $0.5 \mathrm{~m}$ wide mirror was placed at the downstream end of the vessel and 96 PMTs (first prototype version) or 414 PMTs (second version) were placed at the upstream end. The vessel was first evacuated and then filled with neon at atmospheric pressure. The prototype was placed along the old NA48 beam line at CERN.

In the Autumn of 2007 the first prototype version was exposed to a $200 \mathrm{GeV} / c$ negative pion beam. On average 17 PMTs fired per pion event with a time resolution of 70 ps. The Cherenkov angle resolution turned out to be about $50 \mu \mathrm{rad}$. The results have been published in Ref. [4].

In an early 2009 a second version of the RICH prototype was exposed to a positive pion beam of tunable momentum. The average number of hits at high momentum was around 18 per event, with $70 \mu \mathrm{rad}$ Cherenkov angle resolution and $60 \mathrm{ps}$ time resolution. For each momentum point, two data sets were collected, one at the nominal momentum and one at a momentum where a pion has the same velocity as a muon at the nominal momentum. The pion-muon separation was measured in eight momentum points between 15 and $35 \mathrm{GeV} / c$. The total muon suppression factor, weighted with the signal and background spectrum for the final experiment, turned out to be $0.7 \%$. The results have been published in Ref. [5].

\section{Conclusions}

The NA62 RICH detector will be built between 2010 and 2012, in time for the first expected NA62 run at the end of 2012. The strong $R \& D$ program pursued with a prototype and two test beams gives strong confidence on the performances of the final detector.

\section{References}

[1] G. Anelli, et al., Proposal to measure the rare decay $K^{+} \rightarrow \pi^{+} \nu \bar{v}$ at the CERN SPS, CERN-SPSC-2005-013.

[2] F. Anghinolfi, et al., Nucl. Instr. and Meth. A 533 (2004) 183.

[3] G. Haefeli, et al., Nucl. Instr. and Meth. A 560 (2006) 494.

[4] G. Anzivino, et al., Nucl. Instr. and Meth. A 593 (2008) 314.

[5] B. Angelucci, et al., Nucl. Instr. and Meth. A 621 (2010) 205. 